\title{
Water Saving Irrigation Monitoring Network Localization Algorithm Based on RSSI Ranging
}

\author{
Bo Chang ${ }^{1, *}$, Xinrong Zhang ${ }^{2,3}$ and Baoguo $\mathrm{Xv}^{3}$ \\ ${ }^{1}$ Faculty of Electronic Information Engineering, HuaiYin Institute of Techenology, Huaian 223003, China \\ ${ }^{2}$ Faculty of Automation, HuaiYin Institute of Technology, Huaian 223003, China \\ ${ }^{3}$ School of Internet of Things Engineering, JiangNan University, Wuxi 214122, China \\ *Corresponding author
}

\begin{abstract}
In order to know the soil moisture status of farmland irrigation area, improve the quality of production, for the actual demand of water-saving irrigation monitoring node localization, a weighted centroid localization algorithm of water-saving irrigation monitoring network is proposed based on RSSI ranging. This method mainly includes the RSSI ranging, the measurement distance weighted correction, the monitoring node localization and so on. The operator can monitor the change of soil temperature and humidity in real time through the data of the receiving terminal to realize the automatic control of water saving irrigation and the efficient use of water resources. Simulation results show that this method has good positioning effect, can satisfy the positioning requirement for farmland irrigation soil moisture monitoring system. Compared with the conventional LS location algorithm, the proposed localization algorithm has higher accuracy and lower computational complexity.
\end{abstract}

Keywords-water-saving irrigation monitoring; soil moisture; wireless sensor network (WSN); RSSI ranging; weighted centroid algorithms; positioning

\section{INTRODUCTION}

WSN consists of a large number of low-power micro sensor nodes by self-organization and multi hop way[1] which can sense the environmental information monitoring area, data collection and wireless transmission, has become the data processing platform having the development potential, mainly used in military defense, space exploration, environmental monitoring, agriculture and water-saving irrigation and other fields[2].

At present, the positioning mechanism can be divided into two categories, which is Range-Based and Range-Free [3, 4]. RSSI location algorithm is without adding additional measurement equipment $[5,6]$, but is influenced by the effect of signal attenuation and multipath, so the ranging accuracy is low, need to reduce the ranging error by using the methods of repeatedly measuring and iterative refinement [7, 8]. RSSI ranging modeling is based on the RSS distance measurement, and some scholars consider the non-visual environment of the monitoring system, and establish the NLOS path loss model [9]. On the basis of analyzing the $R F$ signal propagation model, the references [10] proposes two kinds of model parameters calculation methods, namely, the least square method and the average switching method, and compares the two models' ranging performance. The references [11] use machine learning method that is statistical and artificial neural network for ranging, and analyzes the factors that affect its performance, which method further reduces the RSSI based ranging error. These positioning algorithm have a certain role in promoting the development of positioning technology, but most of them are large amount of calculation, and are not suitable for the realization of the wireless monitoring system of water saving irrigation in the limited cost and processing capacity.

This paper designs a weighted centroid localization algorithm with low computational complexity, aiming at the existing problems in the localization algorithm, without increasing the hardware equipment and satisfying the positioning precision of the system of water saving irrigation wireless monitoring, gets the distance by measuring the RSSI value, and then uses the weighted distance relative error correction coefficients on the measurement of distance correction. The computation and communication cost of the proposed algorithm is small, which can effectively restrain the Gauss noise, and the positioning accuracy can meet the requirements of most water saving irrigation monitoring system.

\section{Distance MEASUREMENT BEtweEn Nodes BASED ON} RSSI

\section{A. Wireless Signal Propagation Model}

The path loss of wireless signal propagation has great influence on the accuracy of RSSI measurement. The system adopts lognormal distribution model. RSSI values can be expressed as:

$$
P_{R}(d)=P+G-P_{L}(d)
$$

where $P$ is a node transmit power, $G$ is a node antenna gain, $P_{L}$ $(d)$ is the signal strength loss after a distance of $d$. Because:

$$
P_{L}(d)=P_{L}\left(d_{0}\right)+10 n \lg \left(d / d_{0}\right)
$$

where $P_{L}\left(d_{0}\right)$ is the power loss after the reference distance $d_{0}, n$ is the path loss factor, $d_{0}$ is the reference distance, the general value is $1 m$, then:

$$
P_{R}(d)=P_{R}\left(d_{0}\right)-10 n \lg \left(d / d_{0}\right)+X_{\sigma}
$$


where, $P_{R}\left(d_{0}\right)=P+G-P_{L}\left(d_{0}\right)$, is the received signal strength at $d_{0}, X_{\sigma}$ is the Gaussian random variables which mean is 0 and standard deviation is $4-10$. The path loss $P_{L}\left(d_{0}\right)$ after the reference distance $d_{0}$ is:

$$
P_{L}\left(d_{0}\right)=-10 \lg \left[\frac{G_{t} G_{r} \lambda^{2}}{(4 \pi)^{2} d_{0}{ }^{2} L}\right]
$$

where $G_{t}$ (unit: $d B i$ ) is the transmit antenna gain of a node, $G r$ (unit: $d B i$ ) is the receiving antenna gain, $L$ is the system loss coefficient, $\lambda$ (unit: $m$ ) is the $R F$ signal wavelength. Then $P_{R}\left(d_{0}\right)$ can be calculated by the following formula:

$$
P_{R}\left(d_{0}\right)=P+G-P_{L}\left(d_{0}\right)
$$

\section{B. Calculating of Distance between Nodes}

Assuming that the monitoring nodes located in the wireless monitoring area are evenly distributed randomly, the nodes have the same communication radius, and the communication range can be expressed as a regular circular area. By (2), if $d_{0}=1 m$, it can be obtained:

$$
P_{R}(d)=P_{R}(1)-10 n \lg (d)+X_{\sigma}
$$

If enough nodes are uniformly random deployed in the monitoring node communication range, according to the relationship between $P_{R}(d)$ and $d$, it can be assumed that the maximum distance is $d_{\max }$ corresponds to the minimum received signal strength $P_{\text {Rmin }}$, then:

$$
P_{R \min }=P_{R}(1)-10 n \lg \left(d_{\max }\right)+X_{\sigma}
$$

so, we have:

$$
n=\frac{P_{R}(1)-P_{R \min }}{10 \lg \left(d_{\max }\right)}
$$

The $d_{\max }$ is considered to be the communication radius $r$.

\section{Measuring Distance Refinement Method}

That is Error coefficient weighted correction for measuring distance. Considering two dimensional condition, the anchor node is $A_{i}\left(x_{i}, y_{i}\right), i=1,2, \ldots, n$, where $n$ is the number of anchor nodes involved in the calibration calculation, $A_{0}\left(x_{0}, y_{0}\right)$ is the anchor node to be corrected. The actual distance from $A_{0}\left(x_{0}, y_{0}\right)$ to $A_{i}\left(x_{i}, y_{i}\right)$ is marked as $r_{i}, i=1,2, \ldots, n$. The measurement distance from $P_{R}(d)$ is marked as $d_{i}, i=1,2, \ldots, n$. The ranging relative error from $R S S I$ is recorded as:

$$
\mu_{i}=\frac{r_{i}-d_{i}}{d_{i}}
$$

Then the relative error correction coefficient for the weighted distance of the anchor node $A_{i}\left(x_{i}, y_{i}\right)$ is recorded as:

$$
\mu_{W}=\sum_{i=1}^{n} \frac{r_{i}-d_{i}}{d_{i}^{2} \sum_{i=1}^{n} \frac{1}{d_{i}}}
$$

where, $\mu_{W}$ reflects the RSSI measurement error of anchor node. Here taking into account the weight of different $P_{R}(d)$, the greater the distance between the nodes, the greater the distance error caused by the deviation of $P_{R}(d)$, the smaller the weight of the correction coefficient. The correction distance of the anchor node is expressed as:

$$
d_{u i}^{c}=d_{u i}\left(1+\mu_{W}\right)
$$

where, $d_{u i}$ is the measurement distance between the sensor node and the anchor node $R_{i}, d_{u i}^{c}$ is the correction distance between the sensor node and the anchor node $R_{i}, \mu_{W}$ is the weighted ranging relative error correction coefficient for anchor node $R_{i}$.

\section{MONITORING Node LOCALIZATION ALGORITHM}

\section{A. Centroid Localization Algorithm based on RSSI Distance Weighted}

Assuming the unknown location monitoring node $B_{1}$ received the RSSI values of 3 anchor nodes $A_{1}, A_{2}$ and $A_{3}$ within the scope of its communication, these RSSI values are recorded as $R S S I_{1}, R S S I_{2}$ and $R S S I_{3}$, and the path loss exponent $n$ in the monitoring area of node $B_{1}$ has been obtained, considering $R S S I$ value information and distance weighting factors, then the coordinates $(x, y)$ of the unknown position monitoring node $B_{1}$ can be expressed as:

$$
x=\frac{a_{1}^{k} x_{1}+a_{2}^{k} x_{2}+a_{3}^{k} x_{3}}{a_{1}^{k}+a_{2}^{k}+a_{3}^{k}}
$$

$$
y=\frac{a_{1}^{k} y_{1}+a_{2}^{k} y_{2}+a_{3}^{k} y_{3}}{a_{1}^{k}+a_{2}^{k}+a_{3}^{k}}
$$

where, $k$ is a weighted adjustment factor. $d_{1}, d_{2}$ and $d_{3}$ is the measured distances between $B$ and $A_{1}, A_{2}$ and $A_{3}$, respectively, $a_{1}, a_{2}$ and $a_{3}$ is the weighted coefficients in inverse proportion to the corresponding distance measure, respectively, then

$$
a_{1}=\frac{1}{d_{1}}, \quad a_{2}=\frac{1}{d_{2}}, \quad a_{3}=\frac{1}{d_{3}}
$$


In practical application, by controlling the value of $k$, the degree of the weighted correction can be adjusted, so that the positioning system can reach the best state and get the best positioning effect.

\section{B. Coordinate Correction to the Monitoring Node}

Supposing that the anchor node in the network is denoted as $A_{0}\left(x_{0}, y_{0}\right)$, and its position is unknown. Other anchor nodes are denoted as $A_{i}\left(x_{i}, y_{i}\right)$, where $i=1,2, \ldots, n$. Using the distance from $A_{i}\left(x_{i}, y_{i}\right)$ to $A_{0}\left(x_{0}, y_{0}\right)$, the position of $A_{0}$ can be calculated by the weighted positioning algorithm in this paper, and compared with the actual coordinates, we can get the coordinate error. The coordinate error of anchor node $A_{0}\left(x_{0}, y_{0}\right)$ is denoted as:

$$
e_{x 0}=x_{0}-x_{c 0}, \quad e_{y 0}=y_{0}-y_{c 0}
$$

The standard form of the coordinate error of the $i$ th anchor node is:

$$
e_{x i}=x_{i}-x_{c i}, \quad e_{y i}=y_{i}-y_{c i}
$$

Therefore, the weighted coordinate error of the monitoring area is:

$$
\begin{aligned}
& e_{w x}=\sum_{i=1}^{N} \frac{e_{x i}}{d_{i}^{c} \sum_{i=1}^{N} \frac{1}{d_{i}^{c}}} \\
& e_{w y}=\sum_{i=1}^{N} \frac{e_{y i}}{d_{i}^{c} \sum_{i=1}^{N} \frac{1}{d_{i}^{c}}}
\end{aligned}
$$

where, $N$ is the number of anchor nodes involved in the calculation of network location error, $d_{i}^{c}$ is the correction distance of the $i$ th anchor node.

The coordinates of the unknown nodes in the positioning system are corrected by the correction of the regional positioning error coefficient. The coordinates of the unknown node after correction are:

$$
x=x_{c}+e_{w x}, \quad y=y_{c}-e_{w y}
$$

where, $\left(x_{c}, y_{c}\right)$ is the coordinate values of unknown node calculated by the weighted centroid localization algorithm.

\section{SIMULATION AND EXPERIMENTAL}

\section{A. Simulation Results and Analysis}

The location error of the node $i$ in the network is defined as:

$$
E_{a i}=\frac{\sqrt{\left(x_{c i}-x_{i}\right)^{2}+\left(y_{c i}-y_{i}\right)^{2}}}{R}
$$

where, $i=1,2, \ldots, n, N$ is the number of the unknown node, $R$ is the communication radius. $p_{i}=\left[\begin{array}{ll}x_{c i} & y_{c i}\end{array}\right]^{T}$ is the final estimate position of node $i$, and $z_{i}=\left[\begin{array}{ll}x_{i} & y_{i}\end{array}\right]^{T}$ is the true position of node $i$. The average location error $E_{a}$ of the nodes in the network is defined as:

$$
E_{a}=\frac{\sum_{i=1}^{N} \sqrt{\left(x_{c i}-x_{i}\right)^{2}+\left(y_{c i}-y_{i}\right)^{2}}}{N R}
$$

1) Settings for the simulation environment. In order to simulate the random ranging error, the distance between the nodes which is calculated by the actual coordinates of the monitoring node is superimposed on the Gauss noise with standard deviation $\sigma_{f}$, which is used as the RSSI input in the $R F$ model. The expression of the standard deviation $\sigma_{f}$ is:

$$
\sigma_{f}=\frac{R}{R_{i}} \mu_{i}
$$

where, $R$ represents the maximum communication radius of the monitoring node, $R_{i}$ represents the communication distance of the monitoring node, $\mu_{i}$ represents the ranging error. Then controlling the value of $\mu_{i}$ can simulate different ranging errors.

2) Influence of distance measurement error on positioning accuracy. The number of anchor nodes is 20 , the number of nodes is 400 and the simulation results are shown in Figure I. The algorithm $A$ represents the algorithm of this paper, the algorithm $B$ represents the least squares (LS) algorithm, the algorithm $C$ represents the common centroid location algorithm.

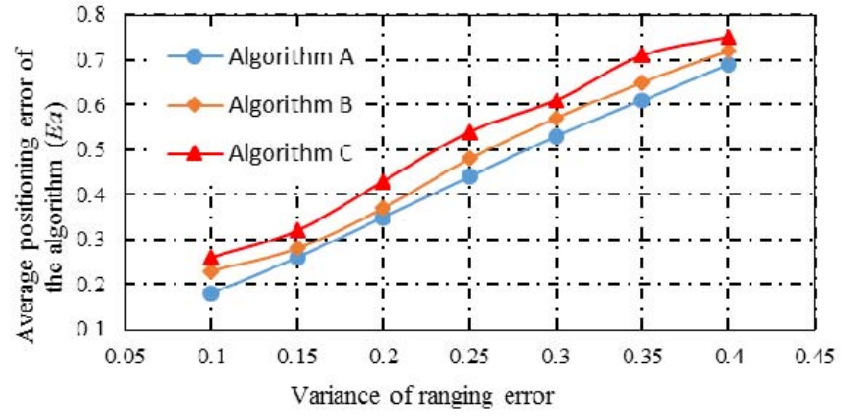

FIGURE I. INFLUENCE OF DIFFERENT RANGING ERRORS ON THE POSITIONING ACCURACY

From Figure I, we can know that the algorithm $C$ is very sensitive to the range error, and with the increase of the distance measurement error, the positioning accuracy of the algorithm is obviously decreased. The algorithm $B$ reduces the bad influence of ranging error on the positioning accuracy, but the effect is not obvious. The algorithm $A$ is very good to 
restrain the error of distance measurement. With the increase of the variance $\sigma_{N}^{2}$ of the ranging error, the positioning accuracy of the three algorithms began to decline, but the positioning accuracy of the algorithm A has been higher than the other two algorithms.

3) Influence of the number of anchor nodes on the Positioning Accuracy. The simulation environment is set to the rectangle area, and the 100 nodes are randomly distributed in the region of $100 m \times 100 m$, the node's communication radius is $40 \mathrm{~m}$. The simulation results are shown in Figure II.

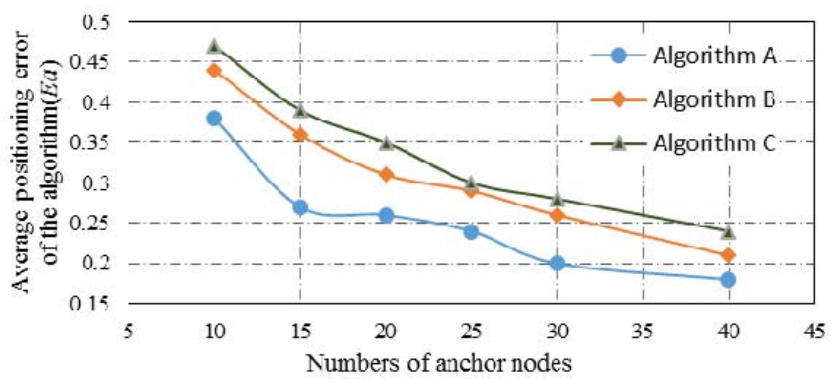

FIGURE II. INFLUENCE OF THE NUMBER OF ANCHOR NODES ON THE POSITIONING ACCURACY

The results from Figure II show that the location error of the algorithm $B$ and algorithm $C$ is larger when the number of anchor nodes is small. Because the algorithm $A$ uses the calibration coefficient of the multiple anchor nodes to correct the measurement distance, it is able to reduce the positioning error caused by the less anchor nodes.

4) Relationship between node communication radius and location accuracy. In the region of $100 \mathrm{~m} \times 100 \mathrm{~m}$, the number of anchor nodes is 10 , the number of nodes is 100 , and the simulation results are shown in Figure III.

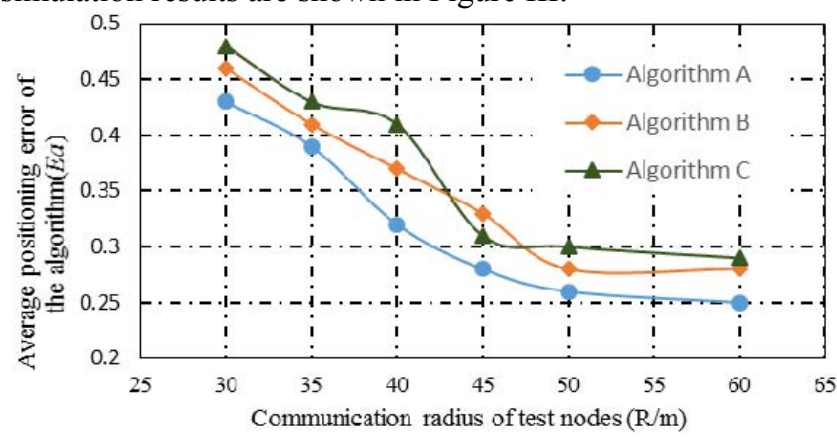

FIGURE III. RELATIONSHIP BETWEEN NODE COMMUNICATION RADIUS AND LOCATION ACCURACY

The results from Figure III show that the positioning accuracy will gradually increase with the increase of the node communication distance, and under the same conditions, the positioning accuracy of the algorithm $A$ is higher than the other two.

\section{B. Experimental Results and Analysis}

In the $20 \mathrm{~m} \times 20 \mathrm{~m}$ area of the laboratory, the experimental system of small wireless sensor network is built using CC2530 nodes. The system has 6 anchor nodes, which are deployed in the test area evenly. In addition, the 10 unknown nodes and one sink node are deployed in the selected position. The distance between the nodes is $15 \mathrm{~m}$, the height from a node to the ground is about $0.5 \mathrm{~m}$, and the data is transmitted every 20 seconds. The mean value of the 50 measurements is taken as the experimental result. According to the experimental data, the experimental results are shown in Table I.

TABLE I. EXPERIMENTAL RESULT

\begin{tabular}{|c|c|c|c|c|}
\hline $\begin{array}{c}\text { Number of } \\
\text { nodes to be } \\
\text { located }\end{array}$ & $\begin{array}{c}\text { Node actual } \\
\text { position }\end{array}$ & $\begin{array}{c}\text { Location after } \\
\text { measurement }\end{array}$ & $\begin{array}{c}\text { Distance } \\
\text { between two } \\
\text { position }\end{array}$ & $\boldsymbol{E}_{\boldsymbol{a}}$ \\
\hline 01 & $(3.0,3.0)$ & $(4.5,3.9)$ & 1.75 & 0.12 \\
\hline 02 & $(3.0,9.0)$ & $(3.6,7.7)$ & 1.43 & 0.10 \\
\hline 03 & $(3.0,12.0)$ & $(5.1,11.7)$ & 2.12 & 0.14 \\
\hline 04 & $(6.0,6.0)$ & $(6.6,7.8)$ & 1.90 & 0.13 \\
\hline 05 & $(6.0,9.0)$ & $(7.4,10.2)$ & 1.84 & 0.12 \\
\hline 06 & $(9.0,6.0)$ & $(8.5,7.6)$ & 1.68 & 0.11 \\
\hline 07 & $(9.0,9.0)$ & $(10.4,9.7)$ & 1.57 & 0.10 \\
\hline 08 & $(12.0,3.0)$ & $(11.3,3.9)$ & 1.14 & 0.08 \\
\hline 09 & $(12.0,9.0)$ & $(10.8,9.5)$ & 1.21 & 0.08 \\
\hline 10 & $(15.0,9.0)$ & $(12.2,10.2)$ & 3.04 & 0.20 \\
\hline
\end{tabular}

By Table I, in the actual monitoring environment, the location error of the algorithm $A$ is 0.20 , the minimum positioning error is 0.08 , and the mean value is 0.118 . Under the same conditions, the average positioning error of the simulation experiment is 0.102 . The actual location result of the algorithm $A$ is slightly lower than that of the simulation. The main reason is that the $R F$ signal between the test nodes is affected by the wall, the equipment and the barrier in the actual test environment, which leads to an increase in the error of RSSI measurement, and the positioning accuracy is impaired.

\section{CONCLUSION}

In this paper, a comprehensive weighted centroid localization algorithm of water-saving irrigation monitoring network is proposed. In the algorithm, the signal propagation attenuation model is applied to measure the RSSI value to obtain the distance information, and the relative error correction coefficient of weighted distance measurement is used to correct the measuring distance. Through the design of a comprehensive weighted centroid localization algorithm, a small amount of computation is used to estimate the coordinates of the monitoring nodes. Simulation results show that this algorithm is less computation and communication costs, can effectively restrain the Gauss noise, the positioning accuracy can meet the requirements of most applications of water-saving irrigation monitoring system, and can obtain the accurate coordinate position information of the network monitoring nodes.

\section{ACKNOWLEDGMENT}

This work was supported by the National Natural Science Foundation of China (Grant No. 21276111), we would like to thank the anonymous reviewers for their perspicacious comments. 


\section{REFERENCES}

[1] F.J. Pierce,T.V. Elliott. Regional and on-farm wireless sensor networks for agricultural systems in Eastern Washington .Computers and Electronics in Agriculture, 2008, 61 (1) :32-43.

[2] Bogena H R, Huisman J A, Oberd rster C, etal. Evaluation of a low-cost soil water content sensor for wireless network applications[J]. Journal of Hydrology, 2007, 344(2): 32-42.

[3] Amitangshu P . Localition algorithms in wireless sensor networks : current approaches and future challenges[J]. Network Protocols and Algorithms, 2010, 2(2):45-74.

[4] Fang J,Cao M,Morse A S,et al.Sequential localization of sensor networks[J].SIAM Journal on Control and Optimization,2009,48(1):321350

[5] Ahn H S, Yu W. Environmental adaptive RSSI based indoor localization[J]. Automation Science and Engineering, 2009, 6(10): 626 -633 .

[6] Li Z, Wang N, Hong T. Radio Path - Loss Modeling For A 2.4 Ghz In Field Wireless Sensor Network[J]. Transactions of the ASABE, 2010, 53(2): $1-10$.

[7] Xu Lei, Shi Weiren. Stepwise refinement location algorithm for wireless sensor network[J]. Chinese Journal of Scientific Instrument, 2008, 29(2): 314-319. (in Chinese with English abstract)

[8] Wang Fubao, Shi Long, Ren Fengyuan. Self-localization systems and algorithms for wireless sensor networks[J]. Journal of Software, 2005, 16(5): 857-868. (in Chinese with English abstract)

[9] H. Ding, Z. Xu. A path loss model for non-line-of-sight ultraviolet multiple scattering channels[J]. EURASIP Journal on Wireless Communication Net-working, 2010: 1-11.

[10] N. Benvenuto, F. Santucci. Comparison between least squares path loss estimation and averaging for handover algorithms[C]. Vehicular Technology Conference on Mobile Technology for the Human Race, Atlanta, GA, 1996:1326-1330.

[11] A. Awad, T. Frunzke. Adaptive distance estimation and localization in WSN using RSSI measures[C]. 10th Euromicro Conference on Digital System Design Architectures, Methods and Tools,Lubeck, 2007; 471478 . 